Pak. j. sci. ind. res. Ser. B: biol. sci. 201154 (2) 59-63

\title{
Emerging Antimicrobial Resistance in Helicobacter pylori Strains Isolated from Gastric Disease Patients in Karachi, Pakistan
}

\author{
Faisal Rasheed ${ }^{a *}$, Adnan Khan ${ }^{a}$, Amber Farooqui ${ }^{a}$, Tanvir Ahmad ${ }^{b}$, Hamid Manzoorc, \\ Syed Shakeel Akhtarc, Mohammed Saeed Quraishy ${ }^{\mathrm{d}}$ and Shahana Urooj Kazmia \\ ${ }^{a}$ Immunology and Infectious Diseases Research Laboratory (IIDRL), Department of Microbiology, \\ University of Karachi, Karachi-75270, Pakistan \\ ${ }^{b}$ Life Science Group, Isotope Application Division (IAD), Pakistan Institute of \\ Nuclear Science and Technology (PINSTECH), Islamabad, Pakistan \\ ${ }^{\mathrm{c}}$ Medical Unit II, Civil Hospital, Dow University of Health Sciences, Karachi, Pakistan \\ ${ }^{\mathrm{d}}$ Department of Surgery, Dow University of Health Sciences, Karachi, Pakistan \\ (received July 28, 2010; revised February 7, 2011; accepted February 28, 2011)
}

\begin{abstract}
In the assessment of the antimicrobial susceptibility of Helicobacter pylori strains isolated from gastric biopsies of patients with gastric diseases against commonly prescribed antibiotics in Pakistan, 31 strains were subjected to antimicrobial susceptibility testing using disk diffusion method against seven antimicrobial agents. Most of the isolates showed resistance to metronidazole (93.5\%), while only $6.5 \%$ isolates were resistant to ofloxacin. The isolates also exhibited variable resistance to other five antibiotics including clindamycin (61.3\%), tetracycline (48.4\%), erythromycin (41.9\%), clarithromycin $(38.7 \%)$ and amoxicillin (29.0\%). Multiple drug resistance in local H. pylori isolates was also observed.
\end{abstract}

Keywords: antimicrobial resistance, H. pylori, culture, disk diffusion

\section{Introduction}

Helicobacter pylori is a gram negative, microaerophillic spiral bacterium that colonizes the stomach of approximately half of the world population (Dunn et al., 1997). Infection with $H$. pylori is associated with chronic gastritis and peptic ulceration and the bacterium is also considered a risk factor for the development of gastric adeno-carcinoma and mucosa-associated lymphoid tissue (MALT) lymphoma (Blaser, 1993; Parsonnet et al., 1991).

H. pylori once acquired persists usually for life unless eradicated by antimicrobial therapy. Treatment regimens against $H$. pylori have been evolving since the early 1990s. Antimicrobial treatment for H. pylori infection is a complicated issue. Currently used regimen for H. pylori treatment includes: two antibiotics and an anti-secretory drug like a proton pump inhibitor, to which bismuth salt can be added (Malfertheiner et al., 2005). Commonly prescribed antibiotics are metronidazole, clarithromycin and amoxicillin (Malfertheiner et al., 2002), while tetracycline is used in rescue therapy (Gisbert and Pajares et al., 2001).

*Author for correspondence; E-mail: frohpl@gmail.com
Antimicrobial resistance in $H$. pylori is a serious problem and it has become an important cause, leading to eradication failure. Information on antimicrobial susceptibility is important for selection of an appropriate treatment regimen. It is necessary to have information about the frequency of resistance to particular antimicrobial agents before the selection of an optimum treatment regimen.

Successful treatment of $H$. pylori infection not only results in eradication of the pathogen, but often also cures and prevents the development of associated diseases (Kuipers, 1997). However, the increasing incidence of resistance to antibiotics is largely responsible for the decline in eradication rates which result in financial losses as well as frustration for the patient. Resistance rates reported vary from $10-90 \%$ for metronidazole, $0-45 \%$ for clarithromycin, $0-33 \%$ for amoxicillin and 5-59\% for tetracycline (Thyagarajan et al., 2003; Boyanova et al., 2002; Al-Qurashi et al., 2001; Kim et al., 2001; Wang et al., 2000). Increasing resistance of $H$. pylori to metronidazole, clarithromycin and amoxicillin have been reported from different parts of the world (Megraud, 2004). The increase in prevalence of antimicrobial resistance in $H$. pylori strains has 
serious implications as, apart from patient compliance, antimicrobial resistance is the most important factor in determining the outcome of antibiotic treatment (Tom et al., 2006). Many studies from Pakistan have emphasized the clinical pattern of gastroduodenal diseases, detection of $H$. pylori infection and comparison of efficacies of different treatment regimens against this pathogen (Yakoob et al., 2004; Khokhar 2002; Abbass et al., 2001). None, to our knowledge, provides information on resistance to commonly used antibiotics for $H$. pylori eradication. High level of resistance to metronidazole $(93.5 \%)$ and the emerging resistance to other antibiotics, currently included or prescribed for treatment of $H$. pylori infections is a matter of great concern in view of the increasing incidence of $H$. pylori infections and their complications. These observations strongly suggest the necessity of re-evaluating the eradication treatment regimens in local setting and taking immediate measures to reduce self prescription practices as well as increasing the patient compliance. The present study was conducted to determine the antibiotic resistance of $H$. pylori isolates against commonly prescribed antibiotics in Pakistani population.

\section{Materials and Methods}

Patients. The study included 110 patients experiencing upper gastrointestinal symptoms including abdominal pain, diarrhoea, nausea and heartburn undergoing upper gastroduodenal endoscopy during December 2005 to June 2006 at Medical Unit II, Civil Hospital, Dow University of Health Sciences, Karachi, Pakistan. Patients with previous $H$. pylori eradication treatment failure or using antibiotics and/or gastric acid inhibitors were excluded from the study. The population consisted of 73 males and 37 females with mean age of 37.7 years and range, 10 to 85 years.

Endoscopy and biopsy sampling. Biopsy specimens were collected from each patient for culture, dipped in $20 \%$ glucose solution (Christopher and Harry, 1997) and transported to the Immunology and Infectious Disease Research Laboratory (IIDRL), Department of Microbiology, University of Karachi, Karachi, Pakistan, in a light-proof insulated box containing ice packs and cultured within an hour.

Culture, growth conditions and identification of H. pylori. Gastric biopsy specimens were chopped and inoculated on Columbia Blood Agar (CM 0331B, Oxoid) containing laked horse blood (SR0048, Oxoid) 7\% and
H. pylori selective supplement Dent (SR 0147E, Oxoid,) containing Vancomycin $5 \mathrm{mg} / 2 \mathrm{~mL}$, Trimethoprim $2.5 \mathrm{mg} / 2 \mathrm{~mL}$, Cefsulodin $2.5 \mathrm{mg} / 2 \mathrm{~mL}$ and Amphotericin B $2.5 \mathrm{mg} / 2 \mathrm{~mL}$. Plates were incubated in a moist microaerophillic atmosphere containing $\mathrm{CO}_{2} 10 \%, \mathrm{O}_{2}$ $5 \%$ and $\mathrm{N}_{2} 85 \%$ by providing Campylobacter gas generating kit (BR 0056A, Oxoid) at $37^{\circ} \mathrm{C}$ for 7 days. After incubation for 3 days, the plates were examined for growth daily. If no growth of $H$. pylori was observed after incubation of 7 days, the plates were discarded. Small rounded colonies were picked and sub-cultured twice to obtain a pure culture. H. pylori strains were identified on the basis of morphology by Gram stain and using oxidase, catalase and rapid urease tests (Enroth et al., 1999).

Antimicrobial susceptibility testing. Seven antimicrobial agents metronidazole, amoxicillin, clarithromycin, erythromycin, tetracycline, clindamycin and ofloxacin were tested against each $H$. pylori isolate using Kirby Bauer disk diffusion procedure (McNulty et al., 2002; Debets-Ossenkopp et al., 1999). Briefly, inoculum from a fresh subculture was made equivalent to $3 \mathrm{McFarland}$ standard and spread using a cotton swab onto blood agar plates. The antibiotic disks were placed and then the plates were incubated at $37^{\circ} \mathrm{C}$ in a microaerophillic environment for 3-4 days. Antibiotic susceptibility was determined by measuring the growth inhibition zone around the disk.

Statistical analysis. Statistical tests were carried out using SPSS software, version 15 (SPSS Inc., Chicago, USA). The group differences were evaluated using the Pearson chi-square for categorical variables; $\mathrm{P}<0.05$ was accepted as statistically significant.

\section{Results and Discussion}

Among 110 symptomatic patients, aged $10-85$ years, 73 were male (66.4\%) and 37 were female (33.6\%). Overall, $32.7 \%$ patients were found to be positive for H. pylori infection by culture. Among the infected patients, there were $37.0 \%$ male and $24.3 \%$ female. No statistical difference was detected between the two sexes in the overall prevalence of $H$. pylori infection $(\mathrm{P}=$ 0.181), (Table 1). As shown in Table 1, 24(34.3\%), $9(32.1 \%)$ and $2(28.6 \%)$ individuals were $H$. pylori positive in the age groups of 19-44, 45-64 years and $65+$ years; the incidence was comparatively higher than that in the age group of 13-18 years with non-statistically significant differences. 
Antimicrobial susceptibility profiles of $31 \mathrm{H}$. pylori isolates were determined by Kirby Bauer disc diffusion procedure. Out of 31 isolates, 25 were from male and 6 from female patients in the age range of 18 to 68 years. Majority of the isolates were found to exhibit high level of resistance to metronidozole $(93.5 \%)$ followed by resistance to clindamycin $(61.3 \%)$, tetracycline (48.4\%), erythromycin (41.9\%), clarithromycin $(38.7 \%)$ and amoxicillin $(29.0 \%)$. However, the resistance to ofloxacin was comparatively low (6.5\%). Resistance to multiple antibiotics was also observed; it was found that double drug resistance pattern for metronidazole and amoxicillin was $12.9 \%$ and that for metronidazole and tetracycline was $9.6 \%$. Triple drug resistance to metronidazole, clindamycin and tetracycline was in $12.9 \%$ of the isolates and $19.3 \%$ multiple resistance was also observed for metronidazole, clindamycin, erythromycin and clarithromycin. Two $(6.5 \%)$ of $H$. pylori isolates were found resistant to all the used antibiotics.

The geographical variation in the antimicrobial resistance of H. pylori is considered to be related to the level of use of the antibiotics in different communities (Graham, 1998). In Pakistan, there is indiscriminate use of antibiotics, especially that of metronidazole, clarithromycin, amoxicillin, erythromycin and tetracycline, for treatment of various infections. In addition, self medication is encouraged by free access to drugs due to un-restricted over-the counter-sale of such antibiotics. This could be a contributing factor for the increased antimicrobial resistance among the $H$. pylori isolates to metronidazole, clarithromycin, tetracycline, clindamycin, erythromycin and amoxicillin, observed in this study. High prevalence of metronidazole resistance might also be due to the frequent use of this antibiotic for other intestinal disorders.

The resistance pattern as reported here is quite similar to what has earlier been reported from India with the exception of tetracycline in which case higher resistance rate $(48.4 \%)$ was observed as compared to $4.2 \%$ (Sengupta et al., 2002) and 28.5\% reported by other investigators (Misra et al., 2006). The resistance rate against the two related antibiotics, clarithromycin and erythromycin, was similar $(38.7 \%$ and $42.0 \%$, respectively), while resistance against ofloxacin was very low $(6.5 \%)$ indicating that it could be used in combination therapy (Schrauwen et al., 2009). Similar to other reports (Falsafi et al., 2004; Torre et al., 2001), multiple drug resistant $H$. pylori isolates were also found which reflects the indiscriminate use of antibiotics in Pakistan.

Resistance to antimicrobial agents among $H$. pylori isolates is prevalent worldwide and it complicates the efforts to eradicate infection. There are reports that prevalence of resistance varies according to the population studied, with resistance to metronidazole ranging from 10 to $90 \%$ and to clarithromycin from 0 to $45 \%$. The associative resistance to metronidazole and clarithromycin is particularly important clinically because these two drugs are frequently prescribed together in a combined therapy. An ideal treatment regimen should be simple, with minimal side effects and most importantly, should consider the local antimicrobial resistance patterns. Accordingly, clinicians could use some help from culture and susceptibility testing before prescribing the best possible treatment

Table 1. H. pylori infection according to sex and age

\begin{tabular}{lllll}
\hline \hline Features & Patients & H. pylori+ $(\%)$ & OR $(95 \% \mathrm{CI})$ & $p$ value \\
\hline $\begin{array}{l}\text { Status } \\
\text { Sex }\end{array}$ & 110 & $36(32.7)$ & & \\
$\quad$ Male & 73 & $27(37.0)$ & $0.54(0.22-1.33)$ & 0.181 \\
$\quad$ Female & 37 & $9(24.3)$ & $1.00(1.00-1.00)$ & 1.000 \\
Age category & & & \\
$\quad$ Adolescent: 13-18 years & 5 & $1(20.0)$ & $1.00(1.00-1.00)$ & 1.000 \\
$\quad$ Adult: 19-44 years & 70 & $24(34.3)$ & $2.08(0.22-19.72)$ & 0.513 \\
$\quad$ Middle aged: 45-64 years & 28 & $9(32.1)$ & $1.89(0.18-19.48)$ & 0.586 \\
$\quad$ Aged: 65+ years & 7 & $2(28.6)$ & $1.60(0.10-24.70)$ & 0.735 \\
\hline \hline
\end{tabular}

Note: ${ }^{\mathrm{a}} \chi^{2}$ test among all groups, OR: odds ratio, CI: confidence interval. 
regimen for the patient depending upon the geographical area.

The antimicrobial resistance pattern among the $H$. pylori isolates was determined first time in Pakistan. The increased resistance could be explained, most probably due to high antibiotics consumption here as compared to other countries. Finally, a high rate of resistance to metronidazole, a considerable resistance to clarithro-mycin, tetracycline, clindamycin, erythromycin, amoxicillin and the least resistance to ofloxacin has been documented. This study provides a basis for future detailed investigations to understand the resistance pattern of $H$. pylori strains isolated from Pakistani population and stresses the need for search of novel antibacterial factors to combat this highly prevalent infection successfully. The recommended treatment regimens in local practice need to be evaluated carefully in the light of studies highlighting the development of resistance in H. pylori isolates. Culture and susceptibility testing is recommended where the first-line and/or the rescue therapy has failed and the patient needs to be cured to reverse the pathophysiological changes associated with $H$. pylori infection.

\section{References}

Abbas, Z., Fareed, R., Baig, M., Khan, T., Shah, M. 2001. Prevalence of histological reflux oesophagitis in $H$. pylori positive patients: effect of density of H. pylori and activity of inflammation. Journal of Pakistan Medical Association, 51: 36-41.

Al-Qurashi, A.R., El-Morsy, F., Al-Quorain, A.A. 2001. Evolution of metronidazole and tetracycline susceptibility pattern in Helicobacter pylori at a hospital in Saudi Arabia. International Journal of Antimicrobial Agents, 17: 233-236.

Blaser, M.J. 1993. Helicobacter pylori: microbiology of a 'slow' bacterial infection. Trends in Microbiology, 1: 255-260.

Boyanova, L., Mentis, A., Gubina, M., Rozynek, E., Gosciniak, G., Kalenic, S., Goral, V., Kupcinskas, L., Kantarceken, B., Aydin, A., Archimandritis, A., Dzierzanowska, D., Vcev, A., Ivanova, K., Marina, M., Mitov, I., Petrov, P., Ozden, A., Popova, M. 2002. The status of antimicrobial resistance of Helicobacter pylori in eastern Europe. Clinical Microbiology and Infection, 8: 388-396.

Christopher, L.C., Harry, L.T.M. 1997. Helicobacter pylori protocols. In: Methods in Molecular Medicine, pp. 17, $2^{\text {nd }}$ edition, Humana Press,
New Jersey, USA.

Debets-Ossenkopp, Y.J., Herscheid, A.J., Pot, R.G., Kuipers, E.J., Kusters, J.G., Vandenbroucke-Grauls, C.M. 1999. Prevalence of Helicobacter pylori resistance to metronidazole, clarithromycin, amoxycillin, tetracycline and trovafloxacin in the Netherlands. Journal of Antimicrobial Chemotherapy, 43: 511-515.

Dunn, B.E., Cohen, H., Blaser, M.J. 1997. Helicobacter pylori. Clinical Microbiology Reviews, 10: 720 741.

Enroth, H., Nyren, O., Engstrand, L. 1999. One stomachone strain: Does Helicobacter pylori strain variation influence disease outcome? Digestive Diseases and Sciences, 44: 102-107.

Falsafi, T., Mobasheri, F., Nariman, F., Najafi, M. 2004. Susceptibilities to different antibiotics of Helicobacter pylori strains isolated from patients at the Pediatric Medical Center of Tehran, Iran. Journal of Clinical Microbiology, 42: 387-389.

Gisbert, J.P., Pajares, J.M. 2001. Helicobacter pylori therapy: first line options and rescue regimen. Digestive Diseases, Basel, Switzerland, 19: 134143.

Graham, D.Y. 1998. Antibiotic resistance in Helicobacter pylori: implications for therapy. Gastroenterology, 115: $1272-1277$.

Khokhar, N. 2002. One week therapy with omeprazole, clarithromycin and amoxicillin for eradication of Helicobacter pylori infection. Journal of College of Physicians and Surgeons Pakistan, 12: 338340.

Kim, J.J., Reddy, R., Lee, M., Kim, J.G., El-Zaatari, F.A., Osato, M.S., Graham, D.Y., Kwon, D.H. 2001. Analysis of metronidazole, clarithromycin and tetracycline resistance of Helicobacter pylori isolates from Korea. Journal of Antimicrobial Chemotherapy, 47: 459-461.

Kuipers, E.J. 1997. Helicobacter pylori and the risk and management of associated diseases: gastritis, ulcer disease, atrophic gastritis and gastric cancer. Alimentary Pharmacology and Therapeutics, 11: 71-88.

Malfertheiner, P., Sipponen, P., Naumann, M., Moayyedi, P., Megraud, F., Xiao, S.D., Sugano, K., Nyren, O. 2005. Helicobacter pylori eradication has the potential to prevent gastric cancer: a state of the art critique. American Journal of Gastroenterology, 100: $2100-2115$.

Malfertheiner, P., Megraud, F., O’Morain, C., Hungin, 
A.P., Jones, R., Axon, A., Graham, D.Y., Tytgat, G. 2002. Current concepts in the management of Helicobacter pylori infection. Alimentary Pharmacology and Therapeutics, 16: 167-180.

McNulty, C., Owen, R., Tompkins, D., Hawtin, P., McColl, K., Price, A., Smith, G., Teare, L. 2002. Helicobacter pylori susceptibility testing by disc diffusion. Journal of Antimicrobial Chemotherapy, 49: 601-609.

Megraud, F. 2004. Helicobacter pylori antibiotic resistance: prevalence, importance, and advances in testing. Gut, 53: 1374-1384.

Misra, R.N., Bhagat, M., Ahmed, N. 2006. Helicobacter pylori in dyspepsia - antibiotic sensitivity and virulence patterns. Medical Journal of Armed Forces of India, 62: 22-26.

Parsonnet, J., Friedman, G.D., Vandersteen, D.P., Chang, Y., Vogelman, J.H., Orentreich, N., Sibley, R.K. 1991. Helicobacter pylori infection and the risk of gastric carcinoma. New England Journal of Medicine, 325: 1127-1131.

Schrauwen, R.W.M., Janssen, M.J.R., de-Boer, W.A. 2009. Seven-day PPI-triple therapy with levofloxacin is very effective for Helicobacter pylori eradication. The Netherlands Journal of Medicine, 67: 96-101.

Sengupta, S., Saraswathi, K., Varaiya, A., Gogate, A. 2002. Helicobacter pylori in duodenal ulcer disease and its eradication. Indian Journal of Medical Microbiology, 20: 163-164.

Thyagarajan, S.P., Ray, P., Das, B.K., Ayyagari, A.,
Khan, A.A., Dharmalingam, S., Rao, U.A., Rajasambandam, P., Ramathilagam, B., Bhasin, D., Sharma, M.P., Naik, S.R., Habibullah, C.M. 2003. Geographical difference in antimicrobial resistance pattern of Helicobacter pylori clinical isolates from Indian patients: Multicentric study. Journal of Gastroenterology and Hepatology, 18: 1373-1378.

Tom, S., Pertti, A., Jukka, R., Karin, W., Henry, N., Lisabeth, B.S., Talley, N.J., Engstrand, L., Agréus, L. 2006. Antimicrobial susceptibility of Helicobacter pylori strains in a random adult Swedish population. Helicobacter, 11: 224-230.

Torre, J., Camorlinga-Ponce, M., Pérez-Pérez, G., Madrazo-De la Garza, A., Dehesa, M., Gonzales-Valencia, G., Munoz, O. 2001. Increasing multidrug resistance in Helicobacter pylori strains isolated from children and adults in Mexico. Journal of Clinical Microbiology, 39: 22677-22680.

Wang, W.H., Wong, B.C., Mukhopadhyay, A.K., Berg, D.E., Cho, C.H., Lai, K.C., Hu, W.H., Fung, F.M., Hui, W.M., Lam, S.K. 2000. High prevalence of Helicobacter pylori infection with dual resistance to metronidazole and clarithromycin in Hong Kong. Alimentary Pharmacology and Therapeutics, 14: 901-910.

Yakoob, J., Jafri, N., Jafri, W., Zaman, S., Bian, L.C., Islam, M., Hussaini, A.S., Zaman, V. 2004. Polymerase chain reaction in the detection of Helicobacter pylori infection. Journal of College of Physicians and Surgeons Pakistan, 14: 153-156. 